\title{
ALGUNAS NOTAS SOBRE LA ECONOFÍSICA
}

Juan E. Trinidad Segovia

UNIVERSIDAD DE ALMERIA

jetrini@ual.es

James M. Chen

MICHIGA STATE UNIVERSITY

chenjame@law.msu.edu

Se denomina con el nombre de Econofísica al área multidisciplinar del conocimiento que intenta combinar la física, las matemáticas y los distintos campos que abarcan la economía. El término fue acuñado por $\mathrm{H}$. Stanley para intentar agrupar al gran número de artículos que los físicos estaban escribiendo en busca de alternativas a diversas cuestiones abiertas en la economía, fundamentalmente en las finanzas de mercado. Este término aparece por primera vez en la conferencia de física que tiene lugar en Calcutta en 1995. No obstante, la conferencia inaugural de este nuevo campo del conocimiento tiene lugar en Budapest en 1998 y es organizada por János Kertész e Imre Kondor.

En cualquier caso, la conexión entre economía, la matemática y física no es en absoluto nueva. Se atribuye a Bernoulli, el descubridor del principio de Bernoulli en hidrodinámica, la introducción del concepto de Utilidad en economía. En 1812, Laplace ya señaló en su trabajo "Essai philoso-phique sur les probabilities" que, pese a que los hechos económicos aparentaban tener una naturaleza impredecible y aleatoria, podían explicarse con leyes bastante simples. Posteriormente en 1835, Quetelet amplió las ideas de Laplace para estudiar la existencia de patrones en datos de naturaleza económica y social. Pocos conocen que el gran economista Fisher tenían formación previa como físico.

No obstante, a veces las cosas funcionan en sentido opuesto, y la primera formulación del Random Walk no es de Eistein, sino del matemático L. Bachelier, que curiosamente desarrolló este modelo para explicar el comportamiento de los precios de los activos financieros allá por el año 1900.

En 1969 el físico Tinbergen ganó el premio Nobel en Economía por el desarrollo y la aplicación de modelos dinámicos en el análisis de los procesos económicos. Ingrao e Israel demostraron que los trabajos de Walras y Pareto sobre el equilibrio estaban basados en el concepto físico de equilibrio mecánico. Uno de los desarrollos más revolucionarios que se han llevado a cabo en la economía llegaron en 1963 de la mano del matemático Mandelbrot (1963), que propuso la utilización de la distribución de Levy para explicar el comportamiento de los precios de los activos financieros en sustitución de la distribución Normal. Este trabajo llamó la atención del laureado economista Eugene Fama (1963), que poco después de la publicación del trabajo de Mandelbrot abrió el debate en el seno de la economía sobre la utilidad de la distribución Normal para modelizar los rendimientos de los activos financieros. Los trabajos de Mandelbrot son todavía hoy por hoy cita habitual en cualquier publicación en este ámbito. De hecho, si sobre los trabajos de Bachelier (1900), Cootner (1964), Samuelson (2004) y Fama (1965) se desarrolló la Teoría del Mercado Eficiente, sobre los trabajos de Mandelbrot (2005), Peters (1989) desarrolló la Teoría del Mercado Fractal como alternativa, donde los concentos de liquidez y memoria juegan un papel crucial.

Por otra parte, frente a aquellos que intentan buscar una distribución para explicar el comportamiento de los precios o los retornos están aquellos que piensan que los mismos son totalmente impredecibles. Las teorías de Poincare sobre la imposibilidad de predicción en sistemas de dinámica no lineal, sobre los que se asentó la teoría del caos, siguen estando de actualidad dentro de la comunidad física y ya se ha extendido al campo de las finanzas. Entre los autores más relevantes están Scheinkman y LeBaron (1989) y Hsieh (1991). 
Algunos de los conceptos introducidos por los físicos en finanzas, tales como el ya comentado de fractalidad o multifractalidad, han sido aceptadas por la mayoría de los economistas y los trabajos entorno al análisis de esta propiedad se han multiplicado a lo largo de la última década. El trabajo de Peters (1989) es la base de muchos realizados con posterioridad y son dignos de mención los trabajos de Bouchaud et al. (2000), Jiang et al. (2019) y Barunik et al. (2012). Sin lugar a dudas, uno de los campos donde más han proliferado los trabajos de los físicos ha sido todo lo relativo a la caracterización de los procesos de variación de los precios de distintos activos financieros, incluyendo el desarrollo de nuevos enfoques. Sin duda, el debate iniciado por Fama en 1963 y todavía sin resolver supuso un aliciente para ellos. Economistas como Lux $(1999 ; 1996 ; 2016)$ y físicos como Stanley (2003) o Masoliver [25] han realizado aportaciones interesantes en este campo ${ }^{1}$.

También ha suscitado mucho interés el estudio de las correlaciones entre las series de precios. En esta línea podemos destacar los trabajos de Mantegna (2000), Stanley $(2000 ; 2015 ; 2018)$ o Bouchaud $(2009 ; 2001)$ entre muchos otros, a los que por cuestiones obvias no puedo citar. Dentro de este campo podemos incluir los trabajos relativos a los conceptos de Memoria en las series, popularizado por Mandelbrot en base a los trabajos del ingeniero Harold Edwin Hurst (1951). Entre los más relevantes de los aparecidos en los últimos años se encuentran los de Barunik y Kristoufek (2010), SánchezGranero et al (2008) o Carbone et al (2004). Sobre la base de que los movimientos Brownianos carecen de memoria, la detección de estos con base en el uso del exponente de Hurst se ha incorporado a las finanzas como el objetivo de analizar la eficiencia de los mercados. En este sentido los trabajos sobre eficiencia han abarcado desde los mercados de acciones hasta las materias primas e incluso las divisas, pasando por diversas clasificaciones de mercados en base a su grado de eficiencia. Podemos citar las contribuciones de Di Matteo et al (2003;2005), Lillo y Farmer (2004), Cajueiro y Tabak (2004) o Sánchez-Granero et al (2008), entre otros.

Sobre este campo en particular presentamos en el monográfico un trabajo de López García y Ramos Requena donde los autores hacen una recopilación de las aplicaciones más interesantes que el exponente de Hurst ha tenido en economía en general y en finanzas en particular.

Carro Barrera presenta un interesantísimo trabajo donde se pretende relacionar la ecuación de Fokker-Planck con el modelo planteado por Black y Scholes, dado que éste último es modelizado mediante una ecuación parcial diferencial estocástica y pueden establecerse similitudes con los procesos estocásticos y de difusión propios de la física.

González García y Mateos Caballero presentan un análisis de la distribución de la riqueza neta en España, incorporando los datos de los activos y pasivos de todas las personas físicas, incluyendo la riqueza derivada de la posesión de acciones en sociedades cotizadas y no cotizadas, con valores actualizados. Se propone un método para deconstruir la Ley de Pareto en componentes que permitiría una modelización realista.

Antunes Ribeiro muestra en su artículo el análisis gráfico de un índice bursátil, realizando la aplicación práctica de la teoría del movimiento de la onda de Elliott del índice bursátil portugués, el PSI 20.

El trabajo de Pinto Ribeiro y otros estudia el comportamiento de las exportaciones agregadas del 2000-2016 para los países que pertenecen a bloques económicos: MERCOSUR, NAFTA y UE. MERCOSUR. Los autores concluyen que la distancia, la crisis posterior al 2008 y el aislamiento de las pequeñas economías insulares perjudican a las exportaciones.

Porto Gómez y otros presentan desarrollan un marco termodinámico basado en modelos de bomba de calor, que se emplea para evaluar el rendimiento de los sistemas de innovación. Este modelo se puede utilizar para evaluar el rendimiento tanto a nivel nacional, como regional, sectorial y empresarial.

\footnotetext{
${ }^{1}$ Por supuesto este es sólo un limitado ejemplo, puesto que es imposible citar todos los trabajos y los autores que han realizado contribuciones significativas en este campo.
} 
Un grupo de colegas de la Universidad de Almería bajo la dirección de Cruz Rambaud proponen un trabajo donde se aplica el EM-index y una nueva versión del factor hiperbólico a la denominada función q-exponencial deformada por la cuantía descuento. Esta función es capaz de explicar casi todos estos estados de impaciencia con respecto al tiempo y la cuantía.

Alfarano y otros analizan la existencia de granularidad en las fluctuaciones del ciclo económico de varias regiones españolas, concretamente de la Comunidad de Madrid, Cataluña, el País Vasco y la Comunidad Valenciana. Los autores concluyen que tanto El País Vasco como la Comunidad Valenciana son economías granulares, mientras que por el contrario la Comunidad de Madrid y Cataluña no lo son.

Por último, concluimos este número especial dedicado a la Econofísica con el trabajo de Jaume Masoliver, al que anteriormente me he referido como uno de los autores más relevantes en este campo del conocimiento. El profesor Masoliver analiza cómo deben compararse los costes futuros con los costes presentes. Generalmente este proceso se realiza mediante una función exponencial con una tasa constante de descuento, cuya elección afecta de forma crítica al valor del futuro, lo que es fundamental para los problemas medioambientales como el calentamiento global. En el trabajo se revisa de forma breve el problema para los profanos en la materia y posteriormente se tiene en cuenta la aleatoriedad en la evolución económica mediante el estudio de tres modelos ampliamente usados para la evolución dinámica de los tipos de interés: Los procesos de Ornstein-Uhlenbeck, Feller y el lognormal. Concluye el autor con una aplicación práctica para una muestra de 9 economías desarrolladas y un periodo de 300 años.

\section{Referencias}

1. Bachelier, L. (1900). Théorie de la spéculation. In Annales scientifiques de l'École normale supérieure Vol. 17, pp. 21-86.

2. Barunik, J., Aste, T. Di Matteo, T. y R. Liu (2012), Understanding the source of multifractality in financial markets, Physica A: Statistical Mechanics and its Applications 391 (17), pp. 4234-4251.

3. Barunik, J. and Kristoufek, L. (2010) On Hurst exponent estimation under heavy-tailed distributions, Physica A: Statistical Mechanics and its Applications 389 (18), pp. 3844-3855

4. Bouchaud, J.P. and M. Potters (2009), Financial Applications of Random Matrix Theory: a short review, arXiv preprint arXiv:0910.1205

5. Bouchaud, J. P., Matacz A. and M. Potters (2001), Leverage effect in financial markets: The retarded volatility model, Physical review letters 87 (22), pp. 228701

6. Bouchaud, J.P., Potters, M. y M. Meyer, M. (2000) Apparent multifractality in financial time series. Eur. Phys. J. B 13, 595-599. https://doi.org/10.1007/s100510050073

7. Cajueiro, D. O. y B. M. Tabak (2004), The Hurst exponent over time: testing the assertion that emerging markets are becoming more efficient, Physica A: Statistical Mechanics and its Applications 336 (3-4), pp. 521-537.

8. Carbone, G. Castelli, H.E. Stanley (2004), Time-dependent Hurst exponent in financial time series. Physica A: Statistical Mechanics and its Applications 344 (1-2), pp. 267-271.

9. Cootner, P. (1964) The random character of stock market prices, Cambridge, Mass., MIT Press,

10.Di Matteo, T., Aste, T. y M.M. (2003) Dacorogna, Scaling behaviors in differently developed markets, Physica A: Statistical Mechanics and its Applications 324 (1-2), pp. 183-188.

11.Di Matteo, T., Aste, T. y M.M. (2005) Dacorogna, Long-term memories of developed and emerging markets: Using the scaling analysis to characterize their stage of development, Journal of Banking \& Finance 29 (4), pp. 827-851.

12.Fama, E. (1963), Mandelbrot and the Stable Paretian Hypothesis, The Journal of Business 36 (4), pp. 420-429

13.Fama, E. (1965), The Behavior of Stock-Market Prices, The Journal of Business 38 (1), pp. 34-105.

14.Gabaix, X., Gopikrishnan, P., Plerou V. and H. E. Stanley (2003), A theory of power-law distributions in financial market fluctuations, Nature 423, pp. 267-270. 
15.Hsieh, D. A. (1991), Chaos and Nonlinear Dynamics: Application to Financial Markets, The journal of finances 46 (5), pp. 1839-1877

16. Hurst, H. E. (1951). Long-term storage capacity of reservoirs. Trans. Amer. Soc. Civil Eng., 116, 770808.

17.Jiang, Z.Q., Xie, W.J., Zhou, W.X. y D. Sornette (2019), Multifractal analysis of financial markets: a review, Rep. Prog. Phys. 82, 125901.

18.Lillo, F., y J.D. Farmer (2004), The Long Memory of the Efficient Market, Studies in Nonlinear Dynamics \& Econometrics, 8(3). doi: https://doi.org/10.2202/1558-3708.1226

19.Lux, T. and M. Marchesi (1999), Scaling and criticality in a stochastic multi-agent model of a financial market, Nature 397, pp 498-500

20.Lux, T. (1996), The stable Paretian hypothesis and the frequency of large returns: an examination of major German stocks, Applied Financial Economics 6 (6), pp. 463-475.

21.Lux, T. and S. Alfarano (2016), Financial power laws: Empirical evidence, models, and mechanisms, Chaos, Solitons \& Fractals 88, Pages 3-18

22. Mandelbrot, B. (1963), The Variation of Certain Speculative Prices, The Journal of Business 36 (4), pp. 394-419

23.Mandelbrot BB. (2005) Fractals and scaling in finance. Springer

24.Mantegna, R.N. and H. E. Stanley (2000), An introduction to Econophysics Correlations and Complexity in Finance, Cambridge University press.

25. Masoliver, J., Montero, M. y G. H. Weiss (2004), A continuous time random walk model for financial distributions, Phys. Rev. E 67, 021112

26.Peters, E. E (1989), Fractal Structure in the Capital Markets, Financial Analysts Journal 45 (4), pp. 32-37.

27.Samuelson, P. (1965) Proof that properly anticipated prices fluctuate randomly, Industrial Management Review, 6, 41-49.

28.Sánchez-Granero, M.A. Balladares, K.A., Ramos-Requena, J.P. y J.E. Trinidad-Segovia (2020), Testing the efficient market hypothesis in Latin American stock markets, Physica A: Statistical Mechanics and its Applications 540, pp. 123082

29.Sánchez -Granero, M.A., Trinidad Segovia, J.E. y García Pérez, J. (2008) Some comments on Hurst exponent and the long memory processes on capital markets, Physica A: Statistical Mechanics and its Applications 387 (22), pp. 5543-5551.

30.Scheinkman, J. A. y B. LeBaron (2011), Nonlinear dynamics and stock returns, The Journal of Business 62 (3), pp. 311-337.

31.Song, D. Tumminello, M. Zhou, W. y R. N. Mantegna (2015), Evolution of worldwide stock markets, correlation structure, and correlation-based graphs, Phys. Rev. E 84, 026108.

32.Stošića, D., Stošića, D., Stošićb, T. y H. E. Stanley (2015), Multifractal properties of price change and volume change of stock market indices, Physica A: Statistical Mechanics and its Applications 428 (15), pp. 46-51

33.Wang, G., Xie, C. y H. E. Stanley (2018), Correlation Structure and Evolution of World Stock Markets: Evidence from Pearson and Partial Correlation-Based Networks, Computational Economics 51 (3), pp 607-635 